\title{
¿Qué nos dice la publicidad sobre los juguetes que promueven habilidades STEM?
}

\author{
Cristian Ferrada \\ adarref@correo.ugr.es \\ https://orcid.org/0000-0003-2678-7334 \\ Universidad de Granada \\ Granada, España \\ Danilo Díaz-Levicoy \\ dddiaz01@hotmail.com \\ https://orcid.org/0000-0001-8371-7899 \\ Universidad Católica del Maule \\ Talca, Chile \\ Eduardo Puraivan \\ epuraivan@uvm.cl \\ https://orcid.org/0000-0003-2134-8922 \\ Universidad de Viña del Mar \\ Viña del Mar, Chile \\ Francisco Silva-Díaz \\ https://orcid.org/0000-0002-7047-3546 \\ Universidad de Granada \\ Granada, España
}

Recibido: 30/04/2020 Aceptado:20/08/2020

\section{Resumen}

En este trabajo se describen los principales resultados de analizar los juguetes con potencialidad de desarrollar habilidades STEM, presente en revistas publicadas previamente a la celebración de las fiestas navideñas en el 2019. Para ello, se realizó un análisis exhaustivo de seis revistas publicitarias de grandes tiendas españolas, identificando un total de 4209 juguetes, de los cuales sólo 160 cumplen con alguno de los criterios para ser considerado un juguete que promueve la Educación STEM. El estudio ha seguido una metodología cualitativa, de nivel descriptivo, por medio de un análisis de contenido, para identificar los Juguetes STEM, su área predominante, las características de los juguetes, su influencia en el género, y el fomento educación STEM. Dentro de los principales hallazgos se destaca la promoción deficiente de juguetes que fomenten la educación STEM, diversos elementos que promueven estereotipos de género entre el público receptor del mensaje, marcando diferencias entre niños/as. Finalmente encontramos las disciplinas de ingeniería y ciencias como las más trabajadas en el logro de los objetivos formulados por los juguetes seleccionados.

Palabras clave: Juegos. Publicidad. Educación STEM. Género. 


\title{
O que a publicidade nos diz sobre brinquedos que promovem habilidades em STEM?
}

\section{Resumo}

Este trabalho descreve os principais resultados da análise de brinquedos com potencial para desenvolver habilidades STEM, presentes em revistas publicadas antes da celebração do Natal de 2019. Para isso, foi realizada uma análise exaustiva de seis revistas de publicidade em grandes lojas. Espanhol, identificando um total de 4209 brinquedos, dos quais apenas 160 cumprem algum dos critérios para serem considerados brinquedos que promovem a educação STEM. O estudo seguiu uma metodologia qualitativa, de nível descritivo, por meio de uma análise de conteúdo, para identificar STEM Toys, sua área predominante, as características dos brinquedos, sua influência no gênero e a promoção da educação STEM. Entre as principais conclusões, destaca-se a pouca promoção de brinquedos que promovem a educação em STEM, vários elementos que promovem estereótipos de gênero entre o público que recebe a mensagem, marcando diferenças entre as meninas e meninos. Por fim, consideramos as disciplinas de engenharia e ciência as mais trabalhadas na consecução dos objetivos formulados pelos brinquedos selecionados.

Palavras chave: Jogos. Publicidade. Educação STEM. Sexo.

\section{What does advertising tell us about toys that promote STEM skills?}

\begin{abstract}
In this work we describe the main results of analyze different toys with the potential to develop STEM skills, presented in magazines published before the celebration of 2019's Christmas. For this purpose, an exhaustive analysis of six advertising magazines in Spanish's large stores was carried out, identifying 4209 toys, of which only 160 meet any of the criteria to be considered a toy that promotes STEM Education. The study has followed a qualitative, descriptive level methodology, through a content analysis, to identify STEM Toys, their predominant area, the characteristics of toys, their influence on gender, and the promotion of STEM education. Among the main findings, the poor promotion of toys that promote STEM education stands out, several elements that promote gender stereotypes among the public receiving the message, marking differences between girls and boys. Finally, we find the engineering and science disciplines as the most worked in achieving the objectives formulated by the selected toys.
\end{abstract}

Keywords: Games. Advertising. STEM education. Gender.

\section{Introducción}

El acrónimo STEM deriva de la aplicación y transversalidad de cuatro disciplinas: Science, Technology, Engineering y Mathematics, las cuales establecen una relación con el fomento, formación, desarrollo de competencias y habilidades en los estudiantes. De manera particular, la ciencia (science) busca la interacción con diferentes fenómenos de la ciencia, química, física y aplicación del método científico. La tecnología (technology) integra las diversas aplicaciones tecnológicas dispuestas en dispositivos móviles, elementos de la electrónica, programación y su aplicación en elementos tales como la robótica, entre otros. La 
ingeniería (engineering) interactúa con construcción, innovación de respuestas a elementos concretos, desarrolla habilidades manuales, elementos de la lógica y creatividad en respuestas a construcciones. Finalmente, las matemáticas (mathematics), proporcionan la base abstracta de los elementos científicos, son aplicadas en la resolución de desafíos, medición de variables, demostración cuantitativa de elementos y desarrollo de la lógica.

Cada una de estas áreas del conocimiento actúan de forma cohesionada, ya sea en las aulas, hogares o instituciones educativas. Su vínculo con la educación se establece al ser la escuela o universidad, el motor de cambio en la sociedad, siendo en estas instituciones donde se forman los futuros agentes que impulsarán el desarrollo científico-tecnológico (Bybee, 2010; Ocaña, 2012).

De esta forma, se identifica la necesidad de preparar a los estudiantes para responder adecuadamente a los diferentes desafíos, tecnológicos, científicos, ingenieriles y matemáticos requeridos por la sociedad (Toma y Meneses-Villagrá, 2019).

Además, existe un consenso general en relación con la alfabetización STEM, el cual no debe verse como un área específica de contenido, sino como un medio para el desarrollo de habilidades cognitivas, conocimientos procedimentales, conceptos y capacidades metacognitivas, las que interactúan para obtener un mayor aprendizaje. Fleer (2013) señala el sentido científico que los estudiantes le dan a los aprendizajes en diversas actividades cotidianas, una vez que comprenden el significado científico-tecnológico. Zollman (2012) considera como objetivo integral de la educación STEM, entregar herramientas para resolver necesidades sociales, producto de los nuevos avances tecnológicos y científicos. Al abordar las necesidades económicas y fortalecer habilidades personales, se logrará contar con ciudadanos satisfechos, productivos y conocedores de los nuevos requerimientos de la sociedad, destacando la necesidad de evolucionar en el aprendizaje.

Otro aspecto importante de este estudio es entregado desde el contexto publicitario, el cual ha adquirido relevancia en nuestra sociedad, especialmente los comerciales televisivos que se apoderan de la imaginación de los niños (Bringué y De Los Ángeles, 2000).

\section{Promoción de la Educación STEM a través de juguetes y publicidad}

El uso de material didáctico es una de las formas más efectivas de fomentar en los estudiantes el despertar de competencias STEM y el desarrollo de la experimentación y 
manipulación de la ciencia. Para Valero-Matas, Callejo, Valero-Oteo y Coca (2017) el desarrollo de una ciencia educativa en las aulas está acompañado de una transmisión de conocimientos, de orden tecnológico, matemático y su posterior aplicación a fenómenos estudiados, idealmente basado en hechos, realidades o experiencias próximas. En tal sentido, la tecnología es vista como un agente de cambio, la que, aproxima a los niños a experimentar y ver la ciencia de forma dinámica, desarrollando aprendizajes basados en la práctica (Siu y Lam 2003; Teo, 2014).

Antes de abordar los juguetes, y algunas de sus características, es necesario definirlos. Según Cabrera (2017) el juguete en educación es visto como una herramienta, con la cual se desarrolla una actividad o experiencia que se disfruta entre los que intervienen en su desarrollo, para lo cual los juguetes representan una manifestación del juego. En relación con los juegos y los juguetes, Hernández (2002) menciona que los juguetes son útiles al servicio del juego infantil, pero que sin el juego no existirían los juguetes. Sin embargo, sin los juguetes los niños seguirán jugando, aunque el juguete se encuentre condicionado al juego.

López-García (2004) analiza el juguete desde su función educativa, establece la relación entre la diversión de manipular un juguete y el enfoque educativo intrínseco que este posee mencionando las propiedades con el área de la Física, ya sea en su composición como en las funciones para lo que fue desarrollado.

Torres, Ramos y Tortoló (2016) consideran que los juguetes tienen un contenido cultural y social, además son esenciales para el desarrollo del niño, quien incorpora a su actividad lúdica el conocimiento y su desarrollo desde la creatividad, imaginación y comunicación.

Según estas concepciones, en la actualidad, los juguetes poseen un doble fin:

- Juguete recreativo: destinado a no poseer una función específica, donde el niño, a través del descubrimiento, encuentra funcionalidad a cada uno de sus componentes.

- Juguete educativo: donde su concepción está definida a un área del sistema escolar, entrega un refuerzo a través de la repetición o metas preestablecidas.

Dentro de esta última categoría encontramos una variante, que establece la interdisciplinariedad en la aplicación del juguete, uniendo la ciencia, ingeniería, tecnología y matemáticas, con el objetivo de conseguir diversos aprendizajes transversales y extrapolando los contenidos curriculares vistos en clases; a estos tipos de juguetes los hemos denominado juguetes STEM. 
Un juguete es STEM no solo por el hecho de ser educativo en su función original, sino que también debe potenciar el desarrollo de las disciplinas científicas (Barrera, 2015). Un juguete STEM integra el juego para lograr despertar la creatividad, habilidades manuales, desarrollo de la memoria y trabajo en grupo en la resolución, de esta manera potencian las habilidades STEM, a través de aspectos lúdicos en ambientes cotidianos. A su vez, Diago, Arnau y González-Calero (2018) y Siu y Lam (2003) establecen el fortalecimiento transversal en la adquisición de competencias elementales para responder a conflictos científicos-tecnológicos y de programación de funciones, en juguetes autónomos de respuesta directa, centrando la articulación de sus aprendizajes en áreas temáticas específicas de las ciencias a través de la exploración.

Es importante establecer que un juguete no siempre es STEM, la relación directa de las cuatro disciplinas que interactúan entre sí hace posible el desarrollo de competencias en estas áreas del conocimiento. Diversos accesorios electrónicos o vinculados con los dispositivos móviles establecen una relación, pero no necesariamente su carácter lúdico las clasifica en el campo de los juguetes STEM (Sullivan y Bers, 2016; Zeidler, 2014).

Por otro lado, al relacionar la publicidad con STEM, las empresas de la industria del juguete hacen esfuerzos para poder captar la atención de los niños, declarando que sus productos facilitan un desarrollo integral de la ciencia, tecnología, matemáticas e ingeniería. Lo anterior, ha tenido un impacto en el mercado por medio de la publicidad, llegando incluso a que empresas como Amazon, que entregan servicios de venta a través de internet, haya creado un apartado en su tienda dedicado a los juegos y juguetes STEM con igualdad de género (Pérez-Ugena, Martínez y Salas 2011), aunque no hay claridad si todos los juguetes que ofrecen, realmente pueden ser considerado así.

Sin embargo, las fechas de celebración especiales para los niños son la oportunidad para que diversas tiendas, a través de afiches, revistas, libros o volantes, publiciten sus promociones, ofertas y descuentos, asociados a juguetes. Para Marti-Pellón y Saunders-Uchoa-Craveiro (2015) la publicidad es vista como un proceso complejo, el cual permite el desarrollo de los países por medio de la sociedad de consumo, donde se pretende fomentar la adquisición de bienes a través de la comunicación social. 


\section{La igualdad de género en la Educación STEM}

Cada vez son más comunes los esfuerzos centrados en la igualdad de roles, orientados a incorporar de forma directa a la mujer, por ejemplo, en diferentes proyectos de Educación STEM. En este sentido, Barragán y Ruiz (2013) analizan la oportunidad que representa la igualdad de género en el mundo científico, resaltando la integración y cooperación que se desarrolla al buscar aprender en un ambiente colaborativo. La integración de las mujeres a equipos de trabajo en áreas STEM, responde a una necesidad de fomentar el trabajo en grupos, el cual ha demostrado que con una mayor diversidad se logra más calidad y mejora la productividad. De esta forma, la elección de carreras STEM apunta directamente a mejorar la integración y participación en la educación, ya que esta formación es vista como uno de los motores en las economías que han logrado un mayor avance en el mundo (Dickson, 2010; Joy, 2000; Turner y Bowen, 1999).

Méndez, Peña e Inda (2012) resaltan que las mujeres presentan una mejor disposición, perseverancia y dominio para completar de forma satisfactoria los estudios de formación científica. Por su parte, Arenas (2011) y Pérez y Gómez (2008) ven, en las nuevas tecnologías, una oportunidad para acercar a las mujeres a un trabajo en campos científicos. Para VázquezCupeiro (2015) los diversos programas que apuntan a tratar el tema de género, poseen un discurso transversal, en los cuales, la estrategia es vista de forma global e innovadora. Spelke (2005) refuerza el concepto de que hombres y mujeres poseen las mismas capacidades en matemática y ciencia, fundamentadas en las bases biológicas de los individuos. De esta forma, la igualdad de género en educación STEM, fomenta el principio de equidad, al momento de realizar un proyecto que trabaje estas áreas disciplinares.

En los últimos años, se ha visto un aumento en la cantidad de mujeres que finalizan carreras STEM, con relación a la década anterior (Seymour, 1995). Sin embargo, aún representan una minoría en comparación a los hombres que finalizan su Educación Superior en profesiones ligadas a los campos científicos. Frente a esto, lograr una igualdad de género en las disciplinas STEM requiere de diferentes esfuerzos, por ejemplo, es necesario fomentar una participación desde edades iniciales en los estudiantes, de esta forma, se entregan igualdad de oportunidades para el logro de las metas iniciales. Atkinson y Mayo (2010) señalan la necesidad de generar programas que apoyen la formación de estudiantes en carreras STEM, así la incorporación de jóvenes en las ciencias aumentará la productividad a corto plazo. En definitiva, 
es necesario fomentar medidas para acercar a las mujeres a estudiar y desarrollar carreras profesionales en entornos tecnológicos-científicos, aumentando el reclutamiento a una edad temprana, como parte de una estrategia importante de inclusión en estas disciplinas (Bybee, 2010; Lagensen, 2007; Toma y Meneses-Villagrá, 2019; Zeidler, 2014).

La Organización para la Cooperación y el Desarrollo Económicos (OCDE, 2015) señala que el $42 \%$ de las mujeres graduadas de carreras de ingeniería o ciencia, trabajan en dichas áreas, por debajo de los hombres (69\%).

Cómo se logra apreciar, la brecha de género no sólo se advierte en el acceso a una carrera de formación científica, sino en el desarrollo profesional, desempeñando trabajos que eventualmente no están vinculados con lo que estudiaron. Mateos-Jiménez, Torres-Martínez y García-Fernández (2018) manifiestan que estas diferencias se han agudizado por dos aspectos: 1) el trato hacia las niñas y adolescentes en edad escolar; y 2) la invisibilidad que algunas mujeres manifiestan en las áreas STEM.

Flores-Espínola (2016) analiza las desigualdades que existen en publicaciones, considerando la variable de género en revistas focalizadas en CTS, proporcionando información sobre la evolución de las mujeres en la producción científica, y revisa las variables metodológicas en el área de inclusión de género. Identificar un total de 2958 artículos desde los inicios de cada revista, hasta el año 2010. Dentro de los resultados es posible apreciar el incremento paulatino de la participación femenina en publicaciones científicas, y el creciente aumento de investigaciones en CTS por parte de las mujeres. Sin embargo, los resultados advierten que desde 1958 al 2010, 1 de cada 4 artículos son escritos por mujeres, y las investigaciones que apuntan al tema de género es solo el $4 \%$.

A nivel internacional, la Organización de las Naciones Unidas para la Educación, la Ciencia y la Cultura (UNESCO, 2016) lanza el proyecto SAGA (STEM and Gender Advancement), apoyado por el Gobierno de Suecia a través de la International Development Cooperation Agency (SIDA), con el objetivo de disminuir de la brecha de género en las áreas STEM, en todos los niveles de educación e investigación, ayudando en el diseño e implementación de transformaciones que acentúan en la igualdad de género en estas disciplinas. De esta forma, el proyecto SAGA busca, a través de sus políticas, mejorar diversos aspectos enfocados a fortalecer la recolección, medición y evaluación de datos por género en actividades STEM. 
De igual forma, SAGA analiza cómo las políticas afectan el equilibrio de género en STEM, aplicando nuevos indicadores que buscan proporcionar material para la creación de diversas políticas basadas en evidencia empíricas medidas para la igualdad de género en Ciencia, Tecnología e Innovación (CTI).

\section{Metodología}

Esta investigación es de tipo cualitativa (Pérez-Serrano, 1994), con un nivel de estudio descriptivo (Hernández, Fernández y Baptista, 2010) y mediante el análisis de contenido (López-Noguero, 2002). Se consideró una muestra intencionada de seis revistas de amplia difusión en el territorio español, de carácter publicitario, promocionadas durante los meses de noviembre y diciembre del año 2019, siendo esta fecha del año una de las de mayores ventas en relación a los artículos destinados a una población infantil. El detalle de las revistas consideradas en el estudio se muestra en la Tabla 1.

Tabla 1.

Datos de las revistas de grandes tiendas españolas sobre juguetes

\begin{tabular}{cll}
\hline Código & \multicolumn{1}{c}{ Tienda } & \multicolumn{1}{c}{ Revista } \\
\hline R1 & Supermercado del Juguete & Navidad Mágica \\
R2 & Al Campo & Sigue la Magia de los Juguetes \\
R3 & Toy Planet & Guía Navidad un Universo de juguetes \\
R4 & Juguetoon & Donde nacen los sueños \\
R5 & Carrefour & Repartir Alegría es Navidad \\
R6 & Juguetilandia & Una navidad para jugar \\
\hline
\end{tabular}

Fuente: elaborado por los autores

Las unidades de análisis han seguido el modelo sobre igualdad de género formulado por la UNESCO (2017), estableciendo 5 unidades de análisis, como se muestra en la Tabla 2. 
Tabla 2.

Descripción de unidades de análisis y categorías

\begin{tabular}{|c|c|c|}
\hline Unidad de análisis & Definición & Categoría \\
\hline $\begin{array}{l}\text { Reconocer la presencia de } \\
\text { juguetes STEM en las } \\
\text { revistas publicitarias }\end{array}$ & $\begin{array}{l}\text { Presencia de juguetes STEM en } \\
\text { revistas de publicidad, involucra o } \\
\text { fomenta a niñas y jóvenes en una } \\
\text { educación STEM, fomentando una } \\
\text { capacitación técnica y de } \\
\text { proyección vocacional en áreas } \\
\text { STEM. }\end{array}$ & $\begin{array}{l}\text { a) Presencia de juguetes } \\
\text { STEM } \\
\text { b) sin presencia de } \\
\text { juguetes STEM }\end{array}$ \\
\hline $\begin{array}{l}\text { Establecer el área STEM } \\
\text { predominante en el juguete }\end{array}$ & $\begin{array}{l}\text { Exactitud del área temática } \\
\text { predominante para desarrollar la } \\
\text { actividad que promueve el juguete } \\
\text { STEM. }\end{array}$ & $\begin{array}{l}\text { a) Ciencia } \\
\text { b) Tecnología } \\
\text { c) Ingeniería } \\
\text { d) Matemáticas }\end{array}$ \\
\hline $\begin{array}{l}\text { Caracterizar si los juguetes } \\
\text { seleccionados como STEM } \\
\text { poseen un carácter } \\
\text { educativo o recreativo. }\end{array}$ & $\begin{array}{l}\text { Identificar la innovación sobre el } \\
\text { accesorio, busca reconocer un } \\
\text { propósito educativo o recreativo en } \\
\text { su transversalidad. }\end{array}$ & $\begin{array}{l}\text { a) Juguetes educati } \\
\text { b) Juguetes recreat }\end{array}$ \\
\hline $\begin{array}{l}\text { Identificar la influencia de } \\
\text { género en la promoción y } \\
\text { difusión del juguete. }\end{array}$ & $\begin{array}{l}\text { Visualizar la presencia de igualdad } \\
\text { de género en juguetes STEM, } \\
\text { mediante la difusión desarrollada } \\
\text { por medio de publicidad en revistas } \\
\text { promocionales. }\end{array}$ & $\begin{array}{l}\text { a) Juguete con influencia } \\
\text { de género. } \\
\text { b) juguete sin influencia } \\
\text { de género. }\end{array}$ \\
\hline $\begin{array}{l}\text { Analizar el fomento de la } \\
\text { educación STEM en los } \\
\text { juguetes }\end{array}$ & $\begin{array}{l}\text { Busca identificar si el juguete } \\
\text { seleccionado fomenta o desarrolla } \\
\text { una educación en base al ejercicio } \\
\text { de habilidades STEM por medio de } \\
\text { la práctica del juego, } \\
\text { específicamente identifica si se } \\
\text { trabajan las cuatro disciplinas de } \\
\text { forma conjunta o de manera } \\
\text { independiente entre ellas. }\end{array}$ & $\begin{array}{lr}\text { a) } & \text { Fomenta } \\
\text { competencias } & \text { STEM } \\
\text { trabajadas íntegramente. } \\
\text { b) fomenta } \\
\text { competencias STEM de } \\
\text { forma aislada. }\end{array}$ \\
\hline
\end{tabular}

Fuente: elaborado por los autores

\section{Resultados}

El análisis consideró 4209 juguetes que se publicitan en las seis revistas de las grandes tiendas españolas. De estos, 160 juguetes son considerados como fomentadores de competencias STEM y en los cuales se estudian según las unidades de análisis recientemente descritas. En la Tabla 3 se muestra la distribución de la cantidad total de juguetes por revista y los que son considerados como STEM. En dicha tabla se observa la baja cantidad de juguetes que promuevan el desarrollo de competencias STEM. 
Tabla 3.

Cantidad de juguetes por revista y cantidad (y porcentaje) de juguetes STEM.

Revista Total juguetes Juguetes STEM

\begin{tabular}{ccc}
\hline R1 & 512 & $1(0,2 \%)$ \\
R2 & 125 & $1(0,8 \%)$ \\
R3 & 640 & $39(6,1 \%)$ \\
R4 & 1261 & $63(5 \%)$ \\
R5 & 832 & $18(2,2 \%)$ \\
R6 & 893 & $32(3,6 \%)$ \\
\hline Total & 4209 & $160(3,8 \%)$ \\
\hline
\end{tabular}

Fuente: elaborado por los autores

En la Figura 1 se muestra uno de los juguetes denominados como STEM, dado que fomenta la vocación científica desde los primeros años de escolaridad (infantil), el cual es transversal y profundiza el trabajo en Educación Primaria (a partir de los 4 años), a través de materiales de laboratorio con los cuales se implementan los talleres científicos.

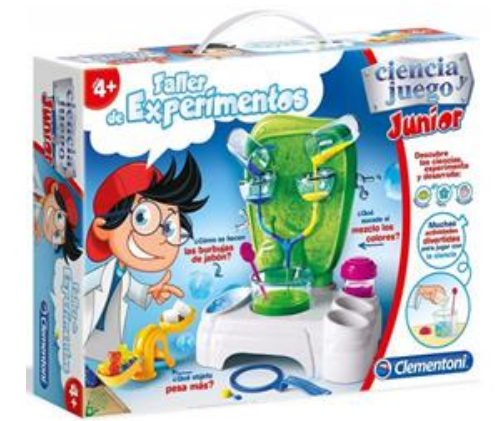

Figura 1. Juguete STEM (R4, p. 35)

En la Tabla 4 se muestra la distribución de los 160 juguetes que promueven el desarrollo de habilidades STEM, según la disciplina considerando el área de conocimiento predominante en su funcionamiento. Los resultados muestran que el principal fomento de habilidades corresponde al de ingeniería (42,87\%), seguido de ciencia (33,1\%), y tecnología (25\%), no se han identificado juguetes que fomenten exclusivamente el área de matemática. 
Tabla 4.

Cantidad y porcentaje de las áreas predominantes en los juguetes STEM

\begin{tabular}{lcc}
\hline Área predominante & Cantidad & Porcentaje \\
\hline Ciencias & 53 & 33,1 \\
Matemáticas & 0 & 0 \\
Tecnología & 40 & 25 \\
Ingeniería & 67 & 41,9 \\
\hline \multicolumn{1}{c}{ Total } & 160 & 100 \\
\hline
\end{tabular}

Fuente: elaborado por los autores

En la Figura 2 se muestra un juguete clasificado como STEM, que incentiva una capacitación técnica con una vocación por la creación, a través del trabajo manipulativo y científico, sin sesgo de género. El juego Geomag focaliza su trabajo en la construcción, a través de sus 120 piezas magnéticas, de diversas formas geométricas. Es posible apreciar dimensiones de longitud, volumen y superficies son necesarios trabajar para la construcción de estructuras. Dentro de sus principales características STEM el magnetismo se desarrolla como una plataforma de actividades exploratorias establecidas por sus elementos, estimula actividades de comprensión numérica, fuerzas magnéticas como eje de construcción, conjuntamente con desafíos ingenieriles de construcción, ya sea para el apoyo y rigidez de las estructuras generadas, siendo el ingenio fundamental para el desarrollo de las tareas propuestas, con lo cual el trabajo de la ingeniería y de las disciplinas adyacentes forman una amalgama de conocimientos puestos en práctica a través del juego.

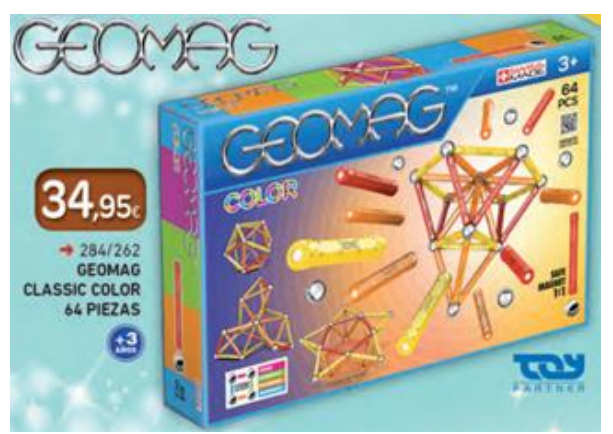

Figura 2. Juguete STEM con énfasis en ingeniería (R2, p. 87) 
En la Tabla 5 mostramos la distribución de los juguetes STEM según su propósito, identificando que la mayoría de ellos tienen un fin recreativo $(54,4 \%)$.

Tabla 5.

Características de los juguetes

\begin{tabular}{ccc}
\hline Características & Cantidad & Porcentaje \\
\hline Recreativos & 87 & 54,4 \\
Educativos & 73 & 45,6 \\
\hline Total & 160 & 100
\end{tabular}

Fuente: elaborado por los autores

En la Figura 3 se muestra el juguete Newton`s Laws, de la serie DiscoveringSTEM, que promueve un rol de género igualitario y sin distinción de público, haciendo hincapié en una formación basada en la educación e innovación, mediante la ciencia y tecnología. Dentro de su descripción se destaca su rol educativo, permitiendo trabajar aspectos de las leyes de movimiento y fuerza de Isacc Newton.

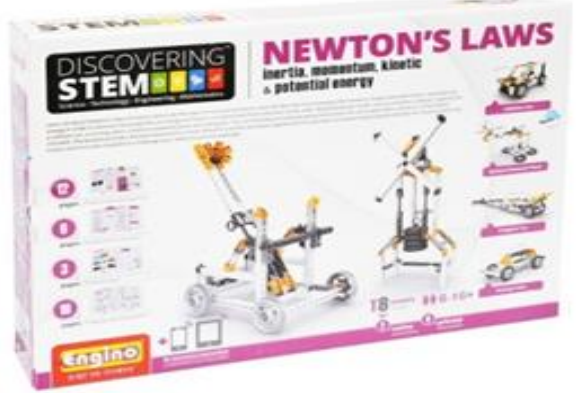

Figura 3. Juguete educativo (R5, p. 64)

Al analizar los juguetes STEM según su disposición al trabajo entre niños y niñas, la Tabla 4 muestra que mayoritariamente no existe una influencia de género $(67,5 \%)$, mientras que $52(32,5 \%)$ juguetes muestran una tendencia hacia la asignación de roles. 
Tabla 6.

Juguetes con y sin influencia en el género

\begin{tabular}{ccc}
\hline Tipo de juguete & Cantidad & Porcentaje \\
\hline Con influencia de género & 52 & 32,5 \\
Sin influencia de género & 108 & 67,5 \\
\hline Total & 160 & 100
\end{tabular}

Fuente: elaborado por los autores

La Figura 4 muestra un juguete con una tendencia publicitaria hacia la desigualdad de género, asignando el rol de médico a un niño, sin mencionar el rol y la participación de las niñas en esta profesión. El juguete denominado Maletín Médico proporciona a los niños diversos accesorios con los cuales experimentan de forma inicial el trabajo en el área de salud, dentro de sus elementos encontramos: estetoscopio, linterna, placa Petri, jeringas, cabestrillos y otros propios de las ciencias. Con estos elementos se puede descubrir elementos de la medicina y de instrumentos que acompañan la función profesional del médico, logrando de esta forma, que los estudiantes puedan mejorar el desarrollo científico exploratorio a través del juego.

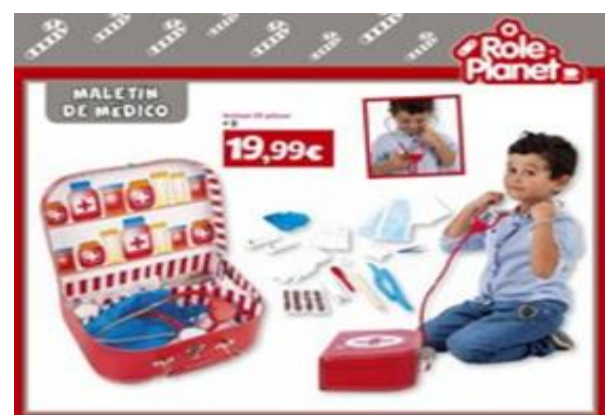

Figura 4. Juguete Maletín médico (R3, p.16).

La Tabla 7 nos muestra que de los 160 juguetes clasificados como STEM, solo un 37,5\% de estos fomenta la adquisición de competencias de STEM en las 4 disciplinas de forma cohesionada, mientras que $62,5 \%$ no fomenta el desarrollo de ciencia, ingeniería, matemática y tecnología de forma armónica y en la sinergia necesaria para poder unir plenamente en una educación STEM con actividades lúdico-recreativas, estando presente el trabajo de las disciplinas de forma parcelada. 
Tabla 7.

Juguetes y el fomento educación STEM

\begin{tabular}{ccc}
\hline Educación STEM & Cantidad & Porcentaje \\
& & \\
\hline Fomenta competencias STEM trabajadas íntegramente & 60 & 37,5 \\
Fomenta competencias STEM de forma aislada & 100 & 62,5 \\
\hline Total & 160 & 100
\end{tabular}

Fuente: elaborado por los autores

En la Figura 5, el fomento de la educación STEM lo observamos mediante el KIT STEM, el cual busca que los niños desarrollen experimentos iniciales en el mundo científico, mediante la manipulación, y guías de trabajo señaladas para tales fines, conociendo de forma directa diversas reacciones químicas y elementos principales de compuestos científicos. En su fin publicitario se fomenta e incentiva la adquisición de este producto, sin discriminar su promoción entre hombre o mujeres, se evidencia la participación de género igualitario en las funciones que promueve la elección de este.

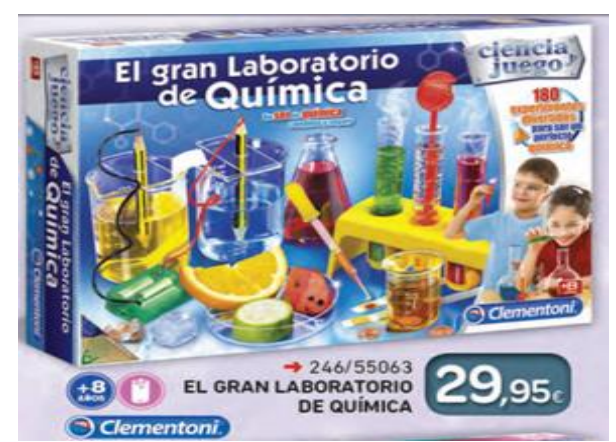

Figura 5. Juguete que fomenta competencias STEM (R6, p. 80)

\section{Conclusión y discusión}

Uno de los principales retos de la educación STEM está en generar espacios para crear un futuro en el cual se pueda aportar desde una óptica distinta del conocimiento, de esta forma la educación con una base científica aporta diversos conocimientos y herramientas necesarias para llegar a este objetivo, aunque parezca el fenómeno STEM un desafío nuevo, este se presenta de la mano con herramientas tecnologías y lúdicas, generando un campo amplio de posibilidades y acceso por parte de los estudiantes, en este escenario encontramos la publicidad y 
específicamente la entregada en momentos en los cuales los niños buscan recibir diversos estímulos concretos, una forma de evaluar la promoción de elementos que fomenten la manipulación, desarrollo y trabajo en una cultura educativa STEM. Para Pérez-Ugena, Martínez y Salas (2011), pese a su importancia, los juguetes cuentan con pocos estudios especializados en la difusión, análisis, propiedades y promoción de juguetes que incremente el trabajo bajo el enfoque STEM, si lo comparamos con otras temáticas, sin identificar las potencialidades que poseen de manera oculta y para los cuales fueron generados. En este estudio se analizaron seis revistas publicitarias, las cuales tienen como objetivo llegar a una gran cantidad de familias y centran su atención en cumplir con expectativas generadas por los niños, de esta forma una elección de un juguete puede resultar beneficioso al poseer este un doble fin, ya sea el educativo como el recreativo. Específicamente, en esta investigación, clasificamos juguetes con o sin un enfoque educativo STEM, obteniendo un total de 160, según el objetivo que persigue el juguete. Estos elementos tienen una especial importancia al establecer su relación de forma directa con la cultura STEM, entendiendo que dichas habilidades al ser trabajadas abren diversas puertas que buscan mejorar el camino que recorrerán los individuos como agentes de cambio y desarrollo académico, aportando una visión complementaria a la educación formal, pero trabajada con herramientas para la consecución de un fin científico tecnológico.

En vista al análisis generado sobre la igualdad de género (Tabla 6) observamos que en dichas revistas, la consecución de este objetivo debe reforzarse en acciones conjuntas: la educación sin sesgo de género debe ser trabajado desde edades tempranas, la concienciación social, la mayor implicación de las empresas en materia de conciliación y las políticas orientadas hacia la igualdad, es fundamental para dar visibilidad a las mujeres que buscan generar una equidad al momento de elegir y trabajar en carreras con vocación en las áreas STEM.

La persistente brecha de género en educación STEM es extrapolada en la promoción de diversos elementos educativos evidenciados en juguetes promocionados en revistas, dentro de las consecuencias encontramos las diversas políticas explícitas e implícitas implementados en varios niveles educativos, sociales, culturales y publicitarios arrastrados en la sociedad. A través del juego, estamos generando estereotipos básicos; es por ello por lo que consideramos que deberían priorizarse en las pantallas de televisión los juguetes considerados neutros para un mejor desarrollo del menor. A tales efectos, sería interesante establecer en relación a la tipología del anuncio, de los valores asociados para difusión. 
Fundamental resulta ver los juguetes y su relación con STEM en innovación, trabajando principios de la lógica necesarios para cumplir su objetivo y de forma paralela despertar el interés científico.

De esta forma encontramos diversos juguetes, los cuales con sus elementos hacen de estos juguetes educativos en consecuencia con su rol transversal y su preponderancia a la recreación, sin embargo esto no determina que puedan generar a través de un juguete STEM un intervención con un fin educativa, ya que estos de por sí, buscan despertar habilidades sistemáticas en los estudiantes, manteniendo un despertar por estas áreas de la investigación recogiendo frecuentemente elementos del magnetismo, cinética, gravedad, aplicados a las áreas STEM como estrategia para lograr el resultado.

Finalmente observamos como la publicidad se enfrenta a un escenario nuevo, para el cual necesariamente tienen que promover el acceso a temprana edad de juguetes con características pedagógicas que despierten la creatividad, imaginación, desarrollo sensorial y el fortalecimiento de las habilidades basado en aprendizajes STEM y aumentar la visibilidad de artículos que potencien un aprendizaje transversal al recreativo, en donde la consecución de la meta sea el trabajo en equipo, exploración de alternativas, aplicación de elementos del método científico y la resolución por medio de las áreas STEM.

\section{Referencias}

ARENAS, M. R. (2011). Brecha digital de género: la mujer y las nuevas tecnologías. Anuario de la Facultad de Derecho, 7(4), 97-125.

ATKINSON, R. D. y MAYO, M. (2010). Refueling the U.S. innovation economy: Fresh approaches to science, technology, engineering and mathematics (STEM) education. Washington, DC: The Information Technology and Innovation Foundation.

BARRAGÁN, R. y RUIZ, E. (2013). Brecha de género e inclusión digital. el potencial de las redes sociales en educación. Profesorado. Revista de Currículum y Formación de Profesorado, 17(1), 309-323.

BARRERA, N. (2015). Uso de la robótica educativa como estrategia didáctica en el aula. Praxis \& Saber, 6(11), 215-234.

BRINGUÉ, X. y DE LOS ÁNGELES, J. (2000). La investigación académica sobre publicidad, televisión y niños: antecedentes y estado de la cuestión. Comunicación y Sociedad, 13(1), 37-70.

BYBEE, R. W. (2010). Advancing STEM Education: A 2020 Vision. Technology and Engineering Teacher, 70(1), 30-35. 
CABRERA, N. A. G. (2017). La importancia del juego como función social a través del desarrollo del ser humano. Ciencia y Actividad Física, 3(2), 30-40.

DIAGO, P. D., ARNAU, D. y GONZÁLEZ-CALERO, J. A. (2018). Elementos de resolución de problemas en primeras edades escolares con Bee-bot. Edma 0-6: Educación Matemática en la Infancia, 7(1), 12-41.

DICKSON, L. (2010). Race and gender differences in college major choice. The ANNALS of the American Academy of Political and Social Science, 627(1), 108-124. doi: $10.1177 / 0002716209348747$

FLEER, M. (2013). Affective imagination in science education: determining the emotional nature of scientific and technological learning of young children. Research in Science Education, 43(5), 2085-2106. doi: 10.1007/s11165-012-9344-8

FLORES-ESPÍNOLA, A. (2016). ¿Los estudios CTS tienen un sexo?: Mujeres y género en la investigación académica. Revista Iberoamericana de Ciencia Tecnología y Sociedad, 11(31), 61-92.

HERNÁNDEZ, R. (2002). El juego en la infancia. Revista Candidus, 4(21), 134-137.

HERNÁNDEZ, R., FERNÁNDEZ, C. y BAPTISTA, P. (2010). Metodología de la investigación. México D.F.: McGraw Hill.

JOY, L. (2000). Do colleges shortchange women? Gender differences in the transition from college to work. American Economic Review, 90(2), 471-475.

LAGESEN, V. A. (2007). The Strength of Numbers: Strategies to Include Women into

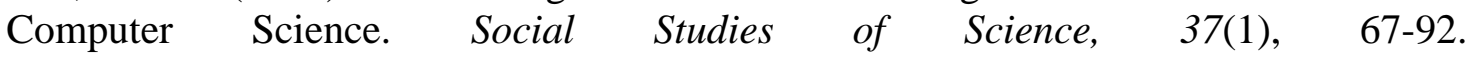
https://doi.org/10.1177/0306312706063788

LÓPEZ-GARCÍA, V. (2004). La física de los juguetes. Revista Eureka sobre Enseñanza y Divulgación de las Ciencias, 1(1), 17-30.

LÓPEZ-NOGUERO, F. (2002). El análisis de contenido como método de investigación. XXI. Revista de Educación, 4, 167-180.

MARTI-PELLÓN, D., y SAUNDERS-UCHOA-CRAVEIRO, P. (2015). Children's Exposure to Advertising on Games Sites in Brazil and Spain. Comunicar, 23(45), 169-177.

MATEOS-JIMÉNEZ, A., TORRES-MARTÍNEZ, B. y GARCÍA-FERNÁNDEZ, B. (2018). Impacto de un relato en las percepciones de racismo y sexismo de escolares de Primaria. Ocnos, 17(2), 55-66. doi: https://doi.org/10.18239/ocnos_2018.17.2.1570

MÉNDEZ, M. C., PEÑA, J. V. e INDA, M. (2012). Creencias de autoeficacia y elección femenina de estudios científico-tecnológicos una revisión teórica de su relación. Teoría de la Educación, 24(1), 81-104.

OCAÑA, G. (2012). Robótica como asignatura en enseñanza secundaria. Resultados de una experiencia educativa. Espiral. Cuadernos del Profesorado, 5(10), 56-64.

OCDE (2015). The ABC of Gender Equality in Education: Aptitude, Behaviour, Confidence. Paris: OCDE.

PÉREZ, E. S. y GÓMEZ, A. (2008). Igualdad y equidad en Ciencia y Tecnología en Iberoamérica. Arbor, 184(733), 785-790. 
PÉREZ-SERRANO, G. (1994). Investigación cualitativa. Retos e interrogantes. I. Métodos. Madrid: La Muralla.

PÉREZ-UGENA, Á., MARTÍNEZ, C. y SALAS, Á. (2011). Los estereotipos de género en la publicidad de los juguetes. Ámbitos. Revista Internacional de Comunicación, 2(20), 217 235.

SEYMOUR, E. (1995). The loss of women from science, mathematics, and engineering undergraduate majors: an explanatory account. Science Education, 79(4), 437-473.

SIU, K., y LAM, M. (2003). Technology education in Hong Kong: International implications for implementing the "Eight Cs" in the early childhood curriculum. Early Childhood Education Journal, 31(2), 143-150.

SPELKE, E. S. (2005). Sex Differences in Intrinsic Aptitude for Mathematics and Science? A Critical Review. American Psychologist, 60(9), 950-958.

SULLIVAN, A. y BERS, M. U. (2016). Robotics in the early childhood classroom: learning outcomes from an 8-week robotics curriculum in pre-kindergarten through second grade. International Journal of Technology and Design Education, 26(1), 3-20.

TEO, T. W. (2014). Hidden Currents in the STEM Pipeline: Insights from the Dyschronous Life Episodes of a Minority Female STEM Teacher. Theory Into Practice, 53(1), 48-54.

TOMA, R. B. y MENESES-VILLAGRÁ, J. A. (2019). Preferencia por contenidos científicos de física o de biología en Educación Primaria: un análisis clúster. Revista Eureka sobre Enseñanza y Divulgación de las Ciencias, 16(1), 1104-16.

TORRES, Y., RAMOS, V. y TORTOLÓ, S. (2016). Los juguetes como medio de desarrollo del niño de la primera infancia. Revista Científica Pedagógica Atenas, 1(33), 110-120.

TURNER, S. E. y BOWEN, W. G. (1999). Choice of major: The changing (unchanging) gender gap. ILR Review, 52(2), 289-313.

UNESCO (2016). Measuring gender equality in science and engineering: the SAGA science, technology and innovation gender objectives list (STI GOL). Working Paper 1. Paris: Autor.

UNESCO (2017). Cracking the code: Girls' and women's education in science, technology, engineering and mathematics (STEM). Paris: Autor.

VALERO-MATAS, J.A., CALLEJO, J., VALERO-OTEO, I. y COCA, J. R. (2017). Análisis de la Elección de Itinerarios Educativos en los Universitarios Españoles. El caso del Campus de Palencia de la Universidad de Valladolid. Multidisciplinary Journal of Educational Research, 7(2), 216- 248.

VÁZQUEZ-CUPEIRO, S. (2015). Ciencia, estereotipos y género: una revisión de los marcos explicativos. Convergencia, 22(68), 177-202.

ZEIDLER, D. (2014). STEM education: A deficit framework for the twenty first century? A socio- cultural socioscientific response. Cultural Studies of Science Education, 11(1), 11-26.

ZOLLMAN, A. (2012). Learning for STEM literacy: STEM literacy for learning. School Science and Mathematics, 112(1), 12-19. 


\section{Autores \\ Cristian Ferrada Ferrada, Universidad de Granada adarref@correo.ugr.es https://orcid.org/0000-0003-2678-7334}

Profesor de Educación General Básica, Mención Educación Matemática, Universidad Católica del Maule. Máster en Didáctica de la Matemática, Universidad de Granada. Doctorando en Ciencias de la Educación, Universidad de Granada. Línea de Investigación:

Didáctica de las Ciencias Experimentales.

Danilo Díaz Levicoy, Universidad Católica del Maule dddiaz01@ hotmail.com https://orcid.org/0000-0001-8371-7899

Profesor de Matemática y Computación (Universidad de los Lagos), Máster en Didáctica de la Matemática (Universidad de Granada) y Doctor en Ciencias de la Educación (Universidad de Granada). Académico de la Facultad de Ciencias Básicas, Universidad Católica del Maule. Línea de Investigación: Didáctica de la Matemática y la Estadística.

Eduardo Puraivan Huenumán, Universidad de Viña del Mar epuraivan@uvm.cl https://orcid.org/0000-0003-2134- 8922

Profesor de matemática (Universidad Católica de Temuco), Ingeniero Civil industrial (universidad Viña del Mar), Magíster en Estadística (Universidad de Valparaíso). Académico de la Escuela de Educación, Universidad de Viña del Mar. Línea de Investigación: Medición

Educacional

Francisco Silva-Díaz, Universidad de Granada fsilva@correo.ugr.es https://orcid.org/0000-0002-7047-3546

Profesor de Educación General Básica, Mención Educación Matemática, Universidad Católica del Maule. Máster en Investigación e Innovación en Currículum y Formación Especialidad Didáctica de las Ciencias Experimentales, Universidad de Granada, España. Doctorando en Ciencias de la Educación, Universidad de Granada. Línea de Investigación:

Didáctica de las Ciencias Experimentales.

Como citar o artigo:

FERRADA, F.; DIAZ-LEVICOY, D.; PURAIVAN, R. Y SILVA-DIAZ, F. ¿Qué nos dice la publicidad sobre los juguetes que promueven habilidades STEM? Revista Paradigma Vol. XLII, Nro. 2, Diciembre de 2021 / 434 - 452.

DOI: $\underline{\text { https://doi.org/10.37618/PARADIGMA.1011-2251.2021.p434-452.id855 }}$ 